\title{
Endoscopic metal stenting for malignant hilar biliary obstruction: an update meta-analysis of unilateral versus bilateral stenting
}

\author{
Fang Yang ${ }^{1}$, Xue-Min Wang ${ }^{2}$, Feng-Fei Xia ${ }^{3}$, Xin-Qiang Han ${ }^{4}$ \\ ${ }^{1}$ Department of Infectious and Liver Disease, Binzhou Medical University Hospital, Binzhou, China \\ ${ }^{2}$ Department of Digestion, Binzhou Medical University Hospital, Binzhou, China \\ ${ }^{3}$ Department of Interventional Vascular Surgery, Binzhou People's Hospital, Binzhou, China \\ ${ }^{4}$ Department of Interventional Vascular Surgery, Binzhou Medical University Hospital, Binzhou, China
}

Videosurgery Miniinv 2021; 16 (3): 472-481

DOI: https://doi.org/10.5114/wiitm.2021.104196

\begin{abstract}
Introduction: Malignant hilar biliary obstruction (MHBO) can arise in patients with malignant hilar hepatobiliary tumors or lymph nodules. Most MHBO patients are not suitable for surgical resection due to the advanced tumor stage. The only palliative treatment available is provided by endoscopic or percutaneous stenting.

Aim: To compare the efficacy of endoscopic unilateral versus bilateral metal stent insertion for treating MHBO. Material and methods: A search of the PubMed, Embase, and Cochrane Library databases identified all relevant studies published until June 2020. The meta-analysis was undertaken using RevMan v5.3.

Results: We identified 154 studies initially, eight of which were used in our meta-analysis. The eight studies included 818 MHBO patients treated using either endoscopic unilateral $(n=396)$ or bilateral $(n=422)$ metal stenting. No significant differences were observed between the two groups in clinical success rate $(O R=2.64 ; p=0.18)$, complication rate $(O R=0.63 ; p=0.46)$, or $O S(H R=1.03 ; p=0.53)$. The bilateral group had a lower stent dysfunction rate without significance $(O R=1.43 ; p=0.09)$. Significantly longer stent patency was observed in the bilateral group $(H R=1.28 ; p=0.01)$. Technical success rate was significantly higher in the unilateral group $(O R=0.26 ; p=0.04)$. Funnel plot analysis indicated an absence of publication bias related to the selected study endpoints.

Conclusions: Our meta-analysis indicated that endoscopic unilateral stenting had a greater technical success rate for MHBO patients than bilateral stenting. However, the bilateral stenting could achieve longer stent patency.
\end{abstract}

Key words: stent, endoscopic, malignant hilar biliary obstruction, unilateral, bilateral.

\section{Introduction}

Malignant hilar biliary obstruction (MHBO) can arise in patients with malignant hilar hepatobiliary tumors or lymph nodules [1-6]. Most MHBO patients are not suitable for surgical resection due to the advanced stage of the tumor [1-6]. The only palliative treatment available is provided by endoscopic or percutaneous stenting.

Metal stents have progressively taken the place of plastic stents, as they provide a higher clinical success rate and better long-term stent patency as compared to plastic stents in those experiencing biliary obstruction $[5,7,8]$. Whether unilateral or bilateral stent insertion is optimal for MHBO treatment remains an open question [9-18]. Some researchers recommend unilateral stenting because they found that unilateral and bilateral stenting provided similar clinical efficacy for patients with MHBO $[6,8$, 12]. However, other researchers have recommended bilateral stenting because they found that bilateral

Address for correspondence

Feng-Fei Xia, Department of Interventional Vascular Surgery, Binzhou People's Hospital, Binzhou, China, e-mail: xff510@126.com 
stenting provided lower re-intervention rates for patients with MHBO [9, 11, 13, 14].

Recently, Ashat et al. [9] performed a meta-analysis comparing unilateral and bilateral metal stenting for $\mathrm{MHBO}$, finding the latter to have a lower rate of re-intervention. However, clinical efficacy might have been influenced by the stenting approach used (percutaneous or endoscopic). Aghaie Meybodi et al. [10] performed a meta-analysis assessing endoscopic unilateral and bilateral metal stent-mediated treatment of $\mathrm{MHBO}$, and they found that endoscopic unilateral and bilateral metal stenting were equally safe and effective. However, they omitted several suitable studies from their metaanalysis $[15,17,18]$

\section{Aim}

We therefore undertook the present meta-analysis to assess relative endoscopic unilateral versus bilateral metal stent insertion for the palliative treatment of MHBO.

\section{Material and methods}

This meta-analysis was conducted according to the Preferred Reporting Items for Systematic Reviews and Meta-Analyses statement. PubMed, Embase, and the Cochrane Library databases were searched for relevant studies published up to June 2020.

\section{Search strategy and study design}

The search strategy adopted used the following search query: $((()(($ cholangiocarcinoma [Title/Abstract]) OR (biliary obstruction [Title/Abstract])) OR (hilar obstruction [Title/Abstract])) OR (biliary malignancy [Title/Abstract])) OR (biliary tract neoplasia [Title/Abstract])) AND ((stent [Title/Abstract]) OR (SEMS [Title/Abstract]))) AND ((unilateral [Title/Abstract]) OR (unilobe [Title/Abstract]))) AND ((bilateral [Title/Abstract]) OR (bilobe [Title/Abstract])).

Inclusion criteria for these studies included: (a) studies comparing outcomes for unilateral and bilateral metal stent insertion for the treatment of $\mathrm{MHBO}$; (b) stents placed via the endoscopic approach, and (c) studies in English.

Studies were excluded if they met any of the following criteria: (a) non-comparative studies; (b) case reports; (c) animal studies; or (d) reviews.

\section{Data extraction and quality assessment}

Two investigators (X.M.W. and X.Q.H.) independently extracted data (authors, publication year, study design, baseline patient characteristics, and treatment information) from each study. Any discrepancies in the extracted data were resolved by the corresponding author.

The Cochrane Risk of Bias tool was used to evaluate randomized controlled trials (RCTs) [19]. Biases were assessed by evaluating the following items: selection, performance, detection, attrition, reporting, and other biases. All non-RCTs were evaluated using the 9-point Newcastle-Ottawa scale [20].

\section{Endpoints of the study}

Analyzed endpoints included rates of technical success, clinical success, complications, stent dysfunction, stent patency, and overall survival (OS). Stent dysfunction rate was the primary endpoint.

\section{Definition of the endpoints}

Successful stent insertion beyond the MHBO site, such that contrast media could pass easily through the stent, was used to define technical success, whereas clinical success was defined as $a \geq 30 \%$ decrease in total bilirubin within 2 weeks after stenting or a $50 \%$ decrease within 4 weeks $[2,3]$. Stent dysfunction was defined by the occurrence of cholangitis or jaundice in affected patients. OS was the time from stent insertion to death.

\section{Statistical analysis}

RevMan v5.3 was used for data analysis. Mantel-Haenszel analyses were used to determine pooled odds ratios (ORs) with 95\% confidence intervals (Cls) for dichotomous variables. Overall survival was analyzed using hazard ratios (HRs) with corresponding 95\% Cls. The $\chi^{2}$ and $l^{2}$ tests were employed to assess heterogeneity, with $R^{2}>50 \%$ being indicative of significant heterogeneity. Analysis proceeded via a random-effects model, while sensitivity and subgroup analyses were employed to assess sources of heterogeneity. Publication bias sources were assessed using funnel plots.

\section{Results}

\section{Study characteristics}

One hundred and fifty-four studies were retrieved by the initial search query. Eight of the 
retrieved studies passed all relevant selection criteria, leading to their inclusion in our meta-analysis (Figure 1). Seven were non-RCTs [11-15, 17, 18] and one was an RCT [16]. Although the Mukai et al. study [14] was an RCT, it compared metal versus plastic stents, not unilateral versus bilateral stenting. The eight studies included $818 \mathrm{MHBO}$ patients treated using either endoscopic unilateral $(n=396$, Photo $1 \mathrm{~A})$ or bilateral $(n=422$, Photo $1 \mathrm{~B})$ metal stenting.

Tables I and II show the details for these eight studies. Endoscopic stenting was performed using the side-by-side method in two studies [11, 17], using the stent-in-stent method in two studies $[12,14]$, and in a further four studies side-by-side and stent-in-stent approaches were both used or the technique used was not defined unequivocally $[13,15,16,18]$. The 5 non-RCTs were evaluated as Newcastle-Ottawa scale 6-8. The risk of bias of the RCT is shown in Figure 2. This RCT had a high risk of blinding of participants and unclear risk of blinding of outcome assessment and other bias.

\section{Technical success}

Technical success rates could be extracted from six of the studies $[11-14,16,18]$. No significant heterogeneity was detected $\left(I^{2}=0 \% ; p=0.91\right.$, Figure $\left.3 \mathrm{~A}\right)$.

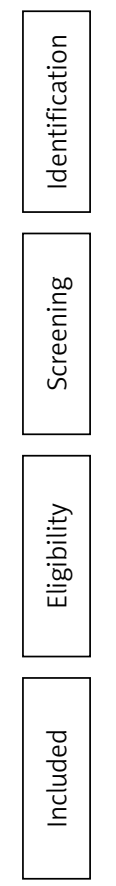

Records identified through database searching $(n=153)$
A significantly higher technical success rate was found in the unilateral group $(\mathrm{OR}=0.26 ; 95 \% \mathrm{Cl}$ : 0.07-0.93, $p=0.04$ ).

\section{Clinical success}

Clinical success rates could be extracted from four of the studies [11, 16-18]. Statistically significant heterogeneity was observed $\left(I^{2}=69 \% ; p=\right.$ 0.02 , Figure $3 \mathrm{~B}$ ). No difference in clinical successful rate was found between groups $(\mathrm{OR}=2.64 ; 95 \% \mathrm{Cl}$ : $0.63-11.06, p=0.18)$. The significant heterogeneity disappeared $\left(I^{2}=17 \% ; p=0.30\right)$ when the Staub et al. study [18] was omitted. After removal, the clinical success rate in the bilateral group was significantly higher $(p=0.009)$.

\section{Complications}

Complication rates could be extracted from five of the studies [11, 12, 15, 16, 18]. Significant heterogeneity was detected $\left(I^{2}=66 \% ; p=0.02\right.$, Figure $\left.3 \mathrm{C}\right)$. No significant difference in complication rates between these groups was found $(\mathrm{OR}=0.63 ; 95 \% \mathrm{Cl}$ : $0.18-2.15, p=0.46$ ). Sensitivity analysis demonstrated that heterogeneity disappeared after omitting the Naitoh et al. study [11] $\left(I^{2}=48 \%\right)$. No differences were detected in complication rates after removing this study $(p=0.91)$.

Additional records identified through other sources $(n=1)$

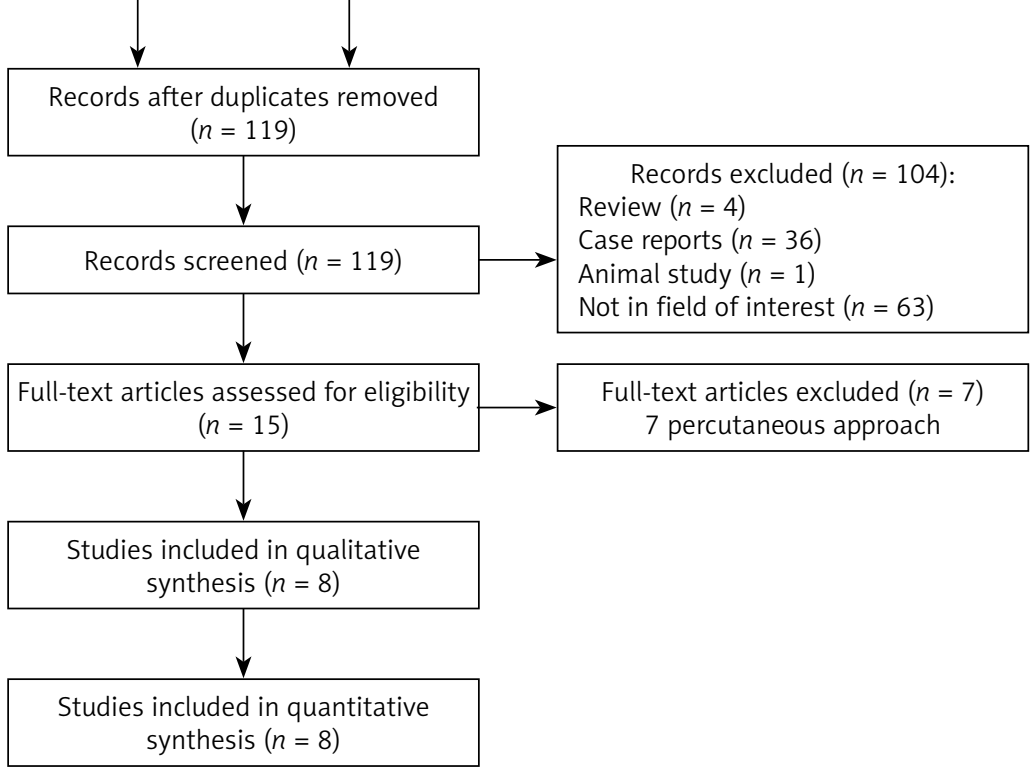

Figure 1. Flowchart detailing the separate stages of the meta-analysis 

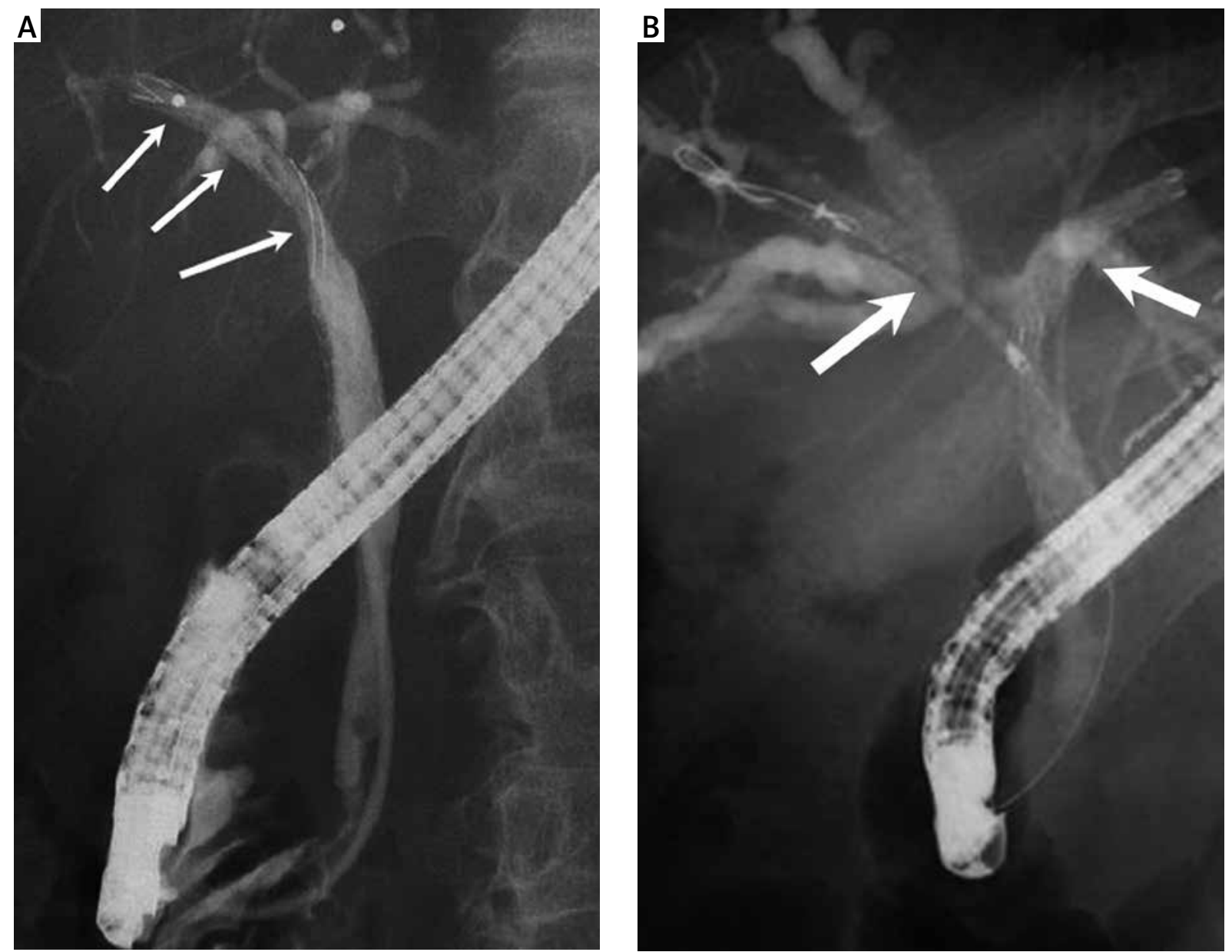

Photo 1. Images for (A) unilateral (arrows) and (B) bilateral (arrows) endoscopic stenting for MHBO

Table I. Characteristics of included studies

\begin{tabular}{|c|c|c|c|c|c|c|c|}
\hline Study/year/country & Study design & Cancer types & Bismuth & Groups & $\begin{array}{c}\text { Sample } \\
\text { size }\end{array}$ & $\begin{array}{c}\text { Age } \\
\text { [years] }\end{array}$ & NOS \\
\hline \multirow[t]{2}{*}{ Naitoh/2009/Japan [11] } & \multirow[t]{2}{*}{ Retrospective } & \multirow[t]{2}{*}{ Multiple } & \multirow[t]{2}{*}{ I-IV } & Unilateral & 17 & 69 & \multirow[t]{2}{*}{8} \\
\hline & & & & Bilateral & 29 & 70 & \\
\hline \multirow[t]{2}{*}{ Iwano/2011/Japan [12] } & \multirow[t]{2}{*}{ Retrospective } & \multirow[t]{2}{*}{ Multiple } & \multirow[t]{2}{*}{ II-IV } & Unilateral & 65 & 71.6 & \multirow[t]{2}{*}{8} \\
\hline & & & & Bilateral & 17 & 66.6 & \\
\hline \multirow[t]{2}{*}{ Liberato/2012/Portugal [13] } & \multirow[t]{2}{*}{ Retrospective } & \multirow[t]{2}{*}{ Cholangiocarcinoma } & \multirow[t]{2}{*}{ II } & Unilateral & 35 & - & \multirow[t]{2}{*}{6} \\
\hline & & & & Bilateral & 42 & - & \\
\hline \multirow[t]{2}{*}{ Mukai /2013/Japan [14] } & \multirow[t]{2}{*}{ Non-RCT } & \multirow[t]{2}{*}{ Multiple } & \multirow[t]{2}{*}{ II-IV } & Unilateral & 14 & - & \multirow[t]{2}{*}{6} \\
\hline & & & & Bilateral & 16 & - & \\
\hline \multirow[t]{2}{*}{ Hatamaru/2017/Japan [15] } & \multirow[t]{2}{*}{ Retrospective } & \multirow[t]{2}{*}{ Multiple } & \multirow[t]{2}{*}{ II-IV } & Unilateral & 52 & 72.5 & \multirow[t]{2}{*}{7} \\
\hline & & & & Bilateral & 27 & 74.4 & \\
\hline \multirow[t]{2}{*}{ Lee/2017/Korea [16] } & \multirow[t]{2}{*}{ RCT } & \multirow[t]{2}{*}{ Multiple } & \multirow[t]{2}{*}{ II-IV } & Unilateral & 66 & 74.1 & \multirow[t]{2}{*}{-} \\
\hline & & & & Bilateral & 67 & 73.5 & \\
\hline \multirow[t]{2}{*}{ Xia/2020/China [17] } & \multirow[t]{2}{*}{ Retrospective } & \multirow[t]{2}{*}{ Multiple } & \multirow[t]{2}{*}{ II-IV } & Unilateral & 97 & 65.4 & \multirow[t]{2}{*}{8} \\
\hline & & & & Bilateral & 87 & 65.5 & \\
\hline \multirow[t]{2}{*}{ Staub/2020/Multicenter [18] } & \multirow[t]{2}{*}{ Retrospective } & Cholangiocarcinoma & I-IV & Unilateral & 50 & 73.1 & 7 \\
\hline & & & & Bilateral & 137 & 72.1 & \\
\hline
\end{tabular}

NOS - Newcastle-Ottawa scale, RCT - randomized controlled trial. 
Table II. Characteristics of treatments

\begin{tabular}{|c|c|c|c|c|c|c|c|c|}
\hline Study & Deployments & Groups & TS & CS & SD & $\begin{array}{c}\text { Patency } \\
\text { [days] }\end{array}$ & $\begin{array}{c}\text { OS } \\
\text { [days] }\end{array}$ & om \\
\hline \multirow{2}{*}{$\begin{array}{l}\text { Naitoh } \\
\text { [11] }\end{array}$} & \multirow[t]{2}{*}{ Side-by-side } & Unilateral & 17/17 (100\%) & 16/17 (94.1\%) & 10/17 (58.8\%) & 210 & 166 & 1/17 (5.9\%) \\
\hline & & Bilateral & 26/29 (89.7\%) & $25 / 26(96.2 \%)$ & 6/26 (23.1\%) & 488 & 205 & $11 / 26(42.3 \%)$ \\
\hline \multirow{2}{*}{$\begin{array}{l}\text { Iwano } \\
{[12]}\end{array}$} & \multirow[t]{2}{*}{ Stent-in-stent } & Unilateral & 60/63 (95.2\%) & Not given & $27 / 65$ (41.5\%) & 133 & 125 & 7/65 (10.8\%) \\
\hline & & Bilateral & $17 / 19(89.5 \%)$ & Not given & $5 / 17$ (29.4\%) & 125 & 126 & 2/17 (11.8\%) \\
\hline \multirow{2}{*}{$\begin{array}{l}\text { Liberato } \\
\text { [13] }\end{array}$} & \multirow[t]{2}{*}{ Both } & Unilateral & $35 / 35$ (100\%) & Not given & $11 / 35$ (31.4\%) & 168 & Not given & Not given \\
\hline & & Bilateral & $42 / 45$ (93.3\%) & Not given & $5 / 42(11.9 \%)$ & 203 & Not given & Not given \\
\hline \multirow{2}{*}{$\begin{array}{l}\text { Mukai } \\
{[14]}\end{array}$} & \multirow[t]{2}{*}{ Stent-in-stent } & Unilateral & Not given & Not given & $4 / 14(28.6 \%)$ & Not given & Not given & Not given \\
\hline & & Bilateral & Not given & Not given & $8 / 16(50 \%)$ & Not given & Not given & Not given \\
\hline \multirow{2}{*}{$\begin{array}{l}\text { Hatama- } \\
\text { ru [15] }\end{array}$} & \multirow[t]{2}{*}{ Not given } & Unilateral & Not given & Not given & $15 / 52(28.8 \%)$ & Not given & Not given & $8 / 52$ (15.4\%) \\
\hline & & Bilateral & Not given & Not given & 9/27 (33.3\%) & Not given & Not given & 3/27 (11.1\%) \\
\hline \multirow[t]{2}{*}{ Lee [16] } & \multirow[t]{2}{*}{ Both } & Unilateral & 66/66 (100\%) & $56 / 66(84.8 \%)$ & $38 / 66(57.6 \%)$ & 139 & 178 & $20 / 66(30.3 \%)$ \\
\hline & & Bilateral & $64 / 67(95.5 \%)$ & 61/64 (95.3\%) & $27 / 64$ (42.2\%) & 252 & 270 & $12 / 64(18.8 \%)$ \\
\hline \multirow[t]{2}{*}{ Xia [17] } & \multirow[t]{2}{*}{ Side-by-side } & Unilateral & Not given & $81 / 97(83.5 \%)$ & 42/97 (43.3\%) & 204 & Not given & Not given \\
\hline & & Bilateral & Not given & 86/87 (98.9\%) & $31 / 87(35.6 \%)$ & 288 & Not given & Not given \\
\hline \multirow{2}{*}{$\begin{array}{l}\text { Staub } \\
{[18]}\end{array}$} & \multirow[t]{2}{*}{ Not given } & Unilateral & $50 / 50(100 \%)$ & Not given & $21 / 50(42 \%)$ & 158 & 249 & $0 / 50(0 \%)$ \\
\hline & & Bilateral & $137 / 137(100 \%)$ & Not given & $60 / 137(43.8 \%)$ & 168 & 207 & $16 / 137(11.7 \%)$ \\
\hline
\end{tabular}

TS - technical success, CS - clinical success, SD - stent dysfunction, OS - overall survival.

\section{Stent dysfunction and patency}

Stent dysfunction rates could be extracted from all eight studies. No statistically significant heterogeneity was detected $\left(I^{2}=39 \% ; p=0.12\right.$, Figure $\left.3 \mathrm{D}\right)$. A lower stent dysfunction rate was observed in the

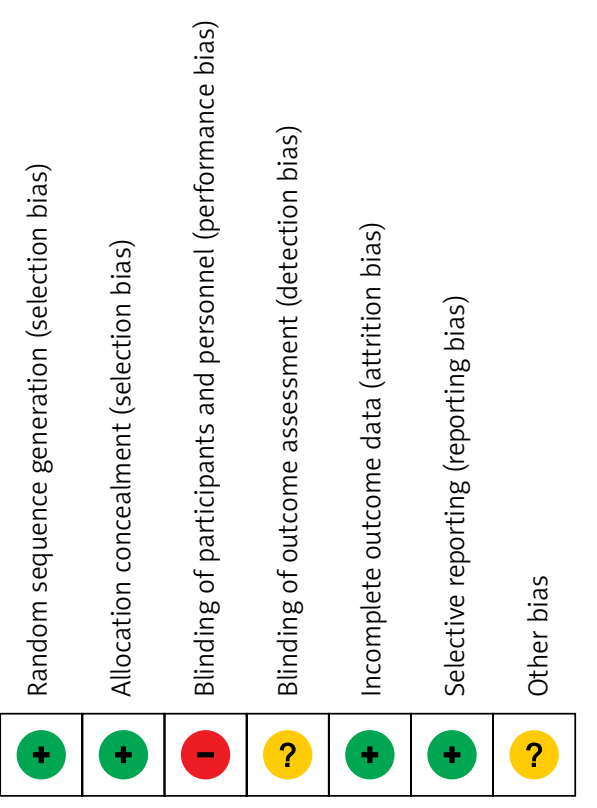

Figure 2. Risk of bias of the included RCT bilateral group, but the difference was non-significant $(\mathrm{OR}=1.43 ; 95 \% \mathrm{Cl}: 0.95-2.17, p=0.09)$.

Stent patency could be extracted from six of the studies [11, 12, 16-18]. Statistically significant heterogeneity was detected $\left(I^{2}=76 \% ; p=0.0007\right.$, Figure $3 \mathrm{E}$ ). Significantly longer stent patency was observed in the bilateral group $(\mathrm{HR}=1.28 ; 95 \% \mathrm{Cl}$ : $1.05-1.56, p=0.01$ ). Sensitivity analyses suggested that removing individual studies had no impact on the detected heterogeneity.

\section{OS}

Overall patient survival could be extracted from four studies $[11,12,16,20]$. Statistically significant heterogeneity was detected $\left(I^{2}=85 \% ; p=0.0002\right.$, Figure $3 \mathrm{~F}$ ). OS did not differ between groups ( $\mathrm{HR}=$ $0.81 ; 95 \% \mathrm{Cl}: 0.42-1.56, p=0.53)$. Sensitivity analysis showed that heterogeneity disappeared after omitting the Staub et al. study [18] $\left(I^{2}=35 \%\right)$. After its removal, OS did not differ between groups $(p=0.61)$.

\section{Subgroup analysis}

Table III shows the pooled stent dysfunction rates for different operative procedures (side-by-side, stent-in-stent, both or unclear) and different disease 
A

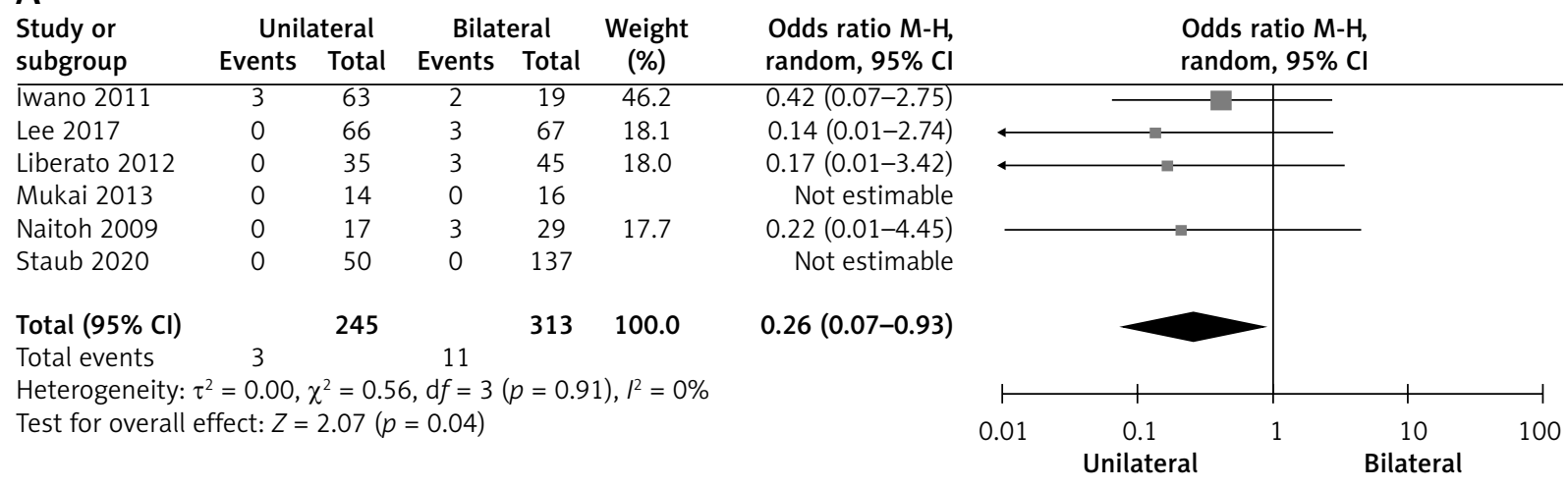

\section{B}

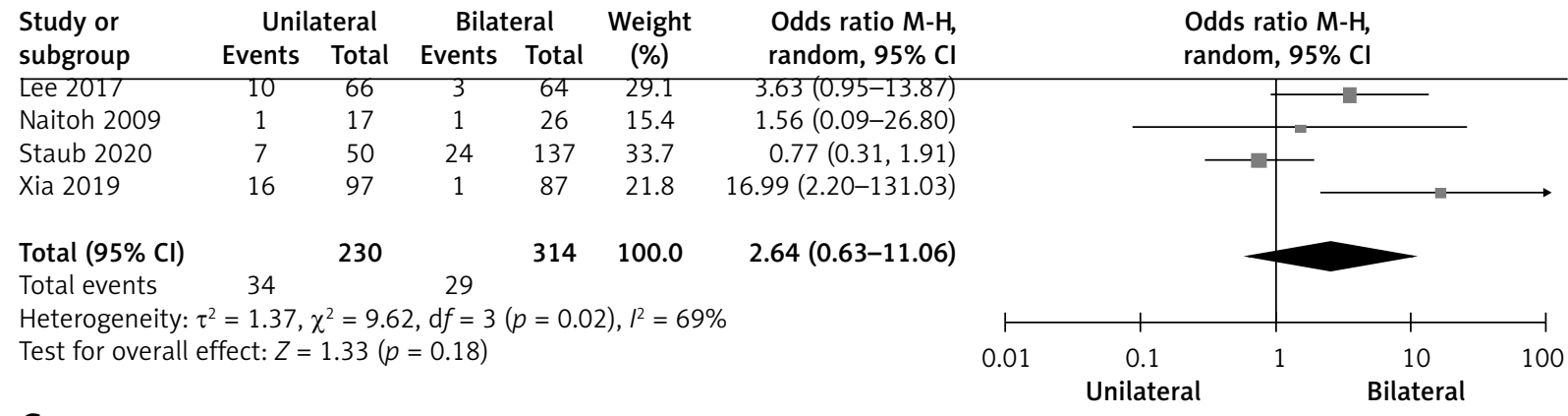

C

\begin{tabular}{lcccccc}
$\begin{array}{l}\text { Study or } \\
\text { subgroup }\end{array}$ & \multicolumn{2}{c}{$\begin{array}{c}\text { Unilateral } \\
\text { Events }\end{array}$} & \multicolumn{2}{c}{$\begin{array}{c}\text { Bilateral } \\
\text { Events }\end{array}$} & $\begin{array}{c}\text { Total } \\
\text { Weight } \\
(\%)\end{array}$ & $\begin{array}{c}\text { Odds ratio M-H, } \\
\text { random, 95\% Cl }\end{array}$ \\
Hatamaru 2017 & 8 & 52 & 3 & 27 & 22.8 & $1.45(0.35-6.00)$ \\
Iwano 2011 & 7 & 65 & 2 & 17 & 20.4 & $0.91(0.17-4.81)$ \\
Lee 2017 & 20 & 66 & 12 & 64 & 28.5 & $1.88(0.83-4.27)$ \\
Naitoh 2009 & 1 & 17 & 11 & 26 & 16.3 & $0.09(0.01-0.74)$ \\
Staub 2020 & 0 & 50 & 16 & 137 & 12.0 & $0.07(0.00-1.24)$ \\
Total $(95 \% \mathrm{Cl})$ & & 250 & & 271 & 100.0 & $0.63(0.18-2.15)$ \\
Total events & 36 & & 44 & & &
\end{tabular}

Heterogeneity: $\tau^{2}=1.22, \chi^{2}=11.92, \mathrm{~d} f=4(p=0.02), I^{2}=66 \%$ Test for overall effect: $Z=0.75(p=0.46)$

D

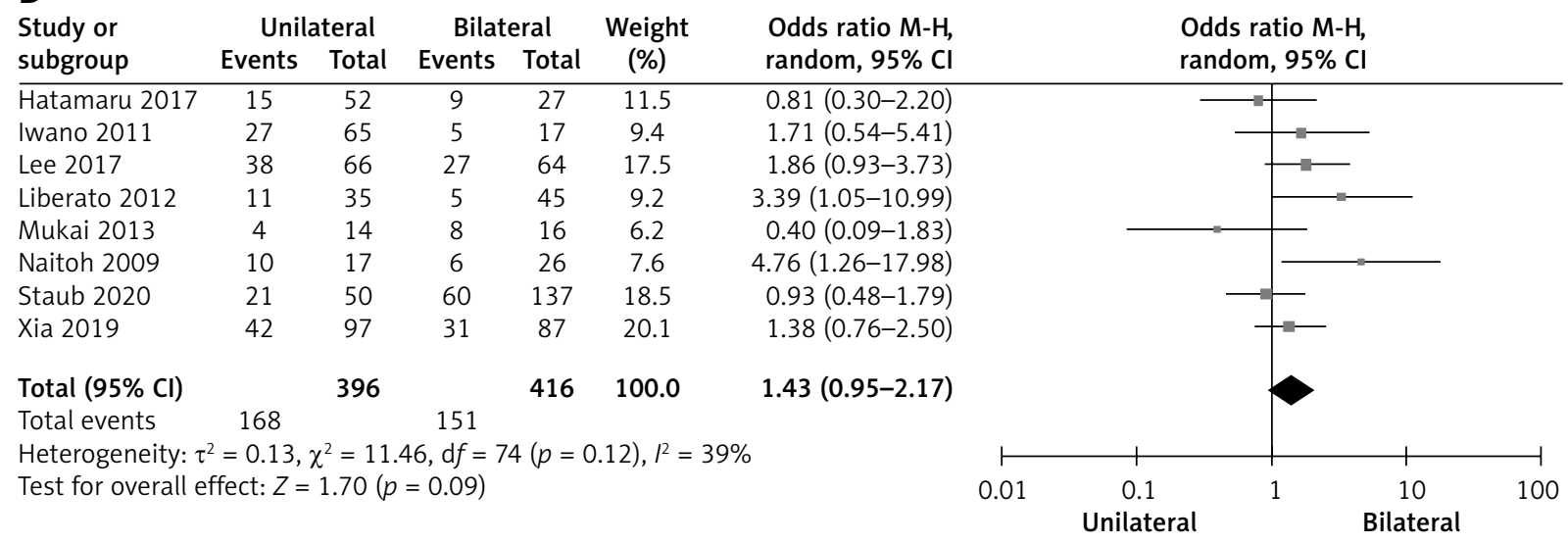

Figure 3. Forest plots detailing comparisons of technical success rates (A), clinical success rates (B), complication rates $(\mathbf{C})$, stent dysfunction rates $(\mathbf{D})$ between the unilateral and bilateral stenting groups 
E

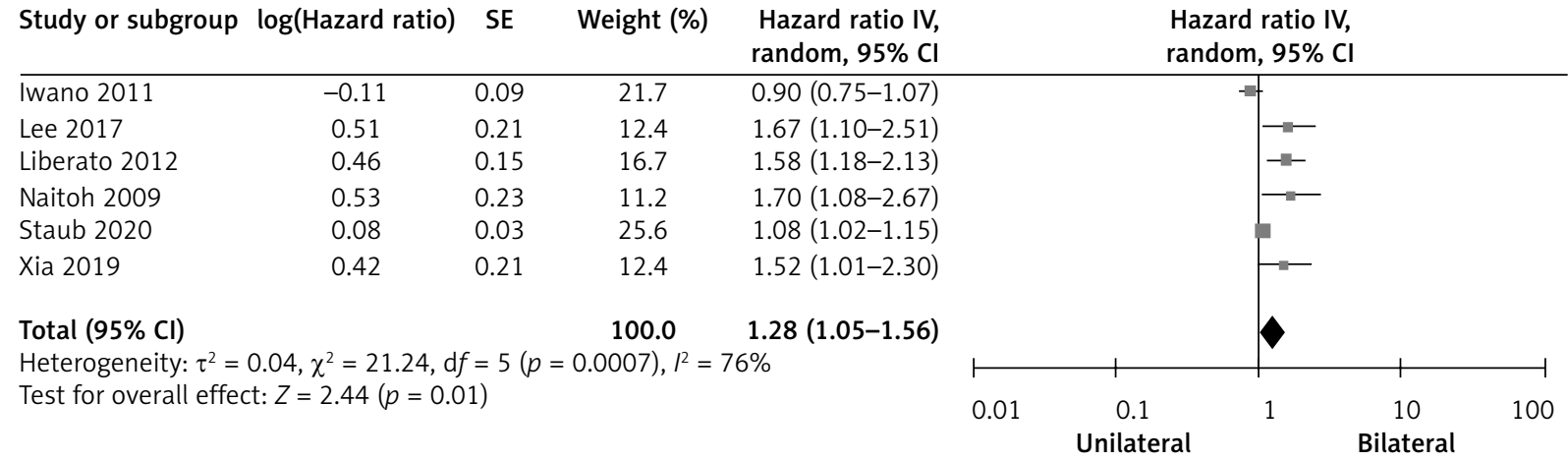

$\mathrm{F}$

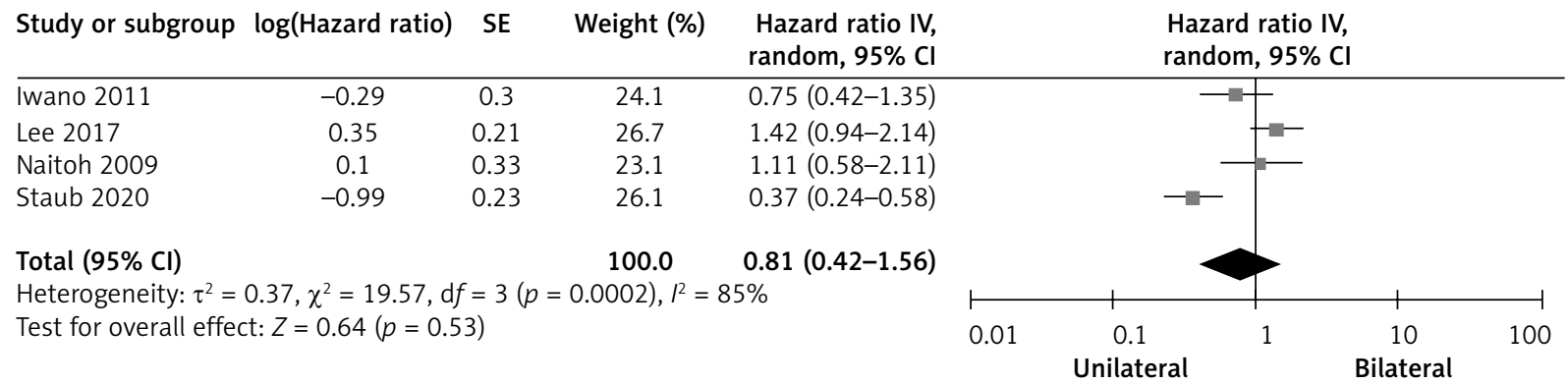

Figure 3. Cont. Forest plots detailing comparisons of stent patency duration $(\mathrm{E})$, and overall survival $(\mathrm{F})$ between the unilateral and bilateral stenting groups

Table III. Meta-analytic pooled stent dysfunction rates based on subgroup analysis

\begin{tabular}{|lcccc|}
\hline Parameter & Number of studies & OR $(95 \% \mathrm{Cl})$ & Favorable & Heterogeneity \\
\hline Total & 8 & $1.43(0.95,2.17), p=0.09$ & - & $R^{2}=39 \%$ \\
\hline Deployments & & & & $R^{2}=64 \%$ \\
\hline Side-by-side & 2 & $2.21(0.68,7.18), p=0.19$ & - & $R^{2}=55 \%$ \\
\hline Stent-in-stent & 2 & $0.99(0.22,3.69), p=0.89$ & - & $R^{2}=45 \%$ \\
\hline Both or unclear & 4 & $1.38(0.79,2.44), p=0.26$ & - & $P^{2}=35 \%$ \\
\hline Diseases & & & - & $R^{2}=72 \%$ \\
\hline Multiple & 6 & $1.44(0.89,2.33), p=0.14$ & - & \\
\hline Cholangiocarcinoma & 2 & $1.61(0.46,5.66), p=0.46$ & & \\
\hline
\end{tabular}

OR - odds ratio.

classifications (multiple or cholangiocarcinoma). All subgroups had equivalent stent dysfunction rates.

\section{Publication bias}

Funnel plot analysis suggested no publication bias relating to the selected study endpoints.

\section{Discussion}

In this meta-analysis, we compared relative clinical efficacy and long-term results of endoscopic unilateral versus bilateral metal stent insertion used for palliative treatment of MHBO. We found a significantly higher technical success rate in the unilateral group. It is generally agreed that unilateral stenting is simpler than bilateral stenting. However, many studies of percutaneous stenting for patients with MHBO indicate similar technical success rates for both groups [6, 21-24]. Compared to the endoscopic approach, percutaneous stenting for MHBO is more straightforward, due to the very short distance from the puncture site to the obstructed site. 
We found a similar initial clinical success rate for both unilateral and bilateral groups $(\mathrm{OR}=2.64$; $p=0.18)$, although significant heterogeneity was present $\left(I^{2}=69 \% ; p=0.02\right)$. However, after correcting for heterogeneity, we found that bilateral stenting demonstrated greater clinical success. This is consistent with the recommendation made by the Asia-Pacific Working Group, that drainage volume should be $>50 \%$ of the entire liver [25]. Our result is different from previous studies of percutaneous stenting for $\mathrm{MHBO}$, which indicated similar clinical success rates in the two groups [6, 21-24]. This finding may result from temporarily utilizing a post-percutaneous stenting biliary drainage catheter after percutaneous stenting [6, 21-24], as the catheter may help drain more bile. By contrast, a drainage catheter is not typically used after endoscopic stenting.

We did not find a difference in complication rates between the two groups. Future studies will need to assess complication rates in this context more rigorously.

Stent dysfunction is a key endpoint in biliary stenting. A previous meta-analysis found endoscopic unilateral and bilateral metal stenting techniques to be comparable in their efficacy and safety [10]. However, three suitable studies $[15,17,18]$ had not been included. When we added these studies, we detected a higher stent dysfunction rate in the unilateral group without significance $(p=0.09)$. Subgroup analyses also demonstrated comparable stent dysfunction rates based on the different deployment approaches and cancer types. However, the pooled stent patency was significant longer in the bilateral group $(p=0.01)$. In this meta-analysis, many studies included many patients who underwent side-by-side bilateral stenting $[11,13,16,17]$. This technique allows drainage to continue even when one stent becomes occluded. These results and findings might indicate that although bilateral stenting could not prevent stent dysfunction, it could prolong the time from stent insertion to dysfunction.

Bilateral stent patency may be influenced by several factors, including type of Bismuth, stent deployment approach (side-by-side versus stent-in-stent), or disease type. This is particularly important for stent deployment, since side-by-side stent insertion creates two drainage routes, allowing drainage to continue even when one stent becomes occluded or blocked. The stent-in-stent approach also requires a larger hilar mesh than the side-by-side deployment strategy, thereby potentially allowing for easier tumor ingrowth [26].

We observed similar overall survival in the two treatment groups, perhaps due to most patients in our meta-analysis being from studies where stenting was used as a post-operative anticancer treatment [16-18]. While such stenting may reduce jaundice in affected patients, it cannot affect the primary tumor directly. Additional anti-cancer treatment is needed to extend stent patency and patient survival significantly [27].

Table IV. Details of previous meta-analyses of unilateral vs. bilateral stenting for MHBO

\begin{tabular}{|c|c|c|c|c|c|c|}
\hline $\begin{array}{l}\text { Authors } \\
\text { [references] }\end{array}$ & $\begin{array}{l}\text { Number } \\
\text { of included } \\
\text { studies }\end{array}$ & Approaches & $\begin{array}{l}\text { Type of } \\
\text { stents }\end{array}$ & $\begin{array}{c}\text { Bilateral } \\
\text { techniques }\end{array}$ & Recommend & Reason \\
\hline Hong et al. [28] & 5 & Endoscopic & $\begin{array}{l}\text { Plastic, } \\
\text { Metal }\end{array}$ & SBS, SIS & Unilateral & Simpler procedure \\
\hline Sawas et al. [8] & 7 & Endoscopic & $\begin{array}{l}\text { Plastic, } \\
\text { Metal }\end{array}$ & SBS, SIS & Unilateral & Simpler procedure \\
\hline Li et al. [29] & 10 & $\begin{array}{l}\text { Endoscopic, } \\
\text { Percutaneous }\end{array}$ & $\begin{array}{l}\text { Plastic, } \\
\text { Metal }\end{array}$ & SBS, SIS & Bilateral & Longer patency \\
\hline Ashat et al. [9] & 9 & $\begin{array}{l}\text { Endoscopic, } \\
\text { Percutaneous }\end{array}$ & Metal & SBS, SIS & Bilateral & $\begin{array}{l}\text { Lower re-intervention } \\
\text { rate }\end{array}$ \\
\hline Fu et al. [30] & 7 & Percutaneous & Metal & SBS, SIS & Unilateral & Simpler procedure \\
\hline Chen et al. [31] & 6 & $\begin{array}{l}\text { Endoscopic, } \\
\text { Percutaneous }\end{array}$ & Metal & SBS & Bilateral & $\begin{array}{l}\text { Lower re-intervention } \\
\text { rate }\end{array}$ \\
\hline $\begin{array}{l}\text { Aghaie Meybodi } \\
\text { et al. [10] }\end{array}$ & 5 & Endoscopic & Metal & SBS, SIS & Unilateral & Simpler procedure \\
\hline
\end{tabular}

SBS - side-by-side, SIS - stent-in-stent. 
Table IV shows previous meta-analyses of unilateral vs. bilateral stenting for MHBO [8-10, 28-31] From such work, it is clear that it remains controversial whether unilateral or bilateral stenting is the method of choice for patients with MHBO. Many factors, including stent types, stenting approaches, and bilateral stenting techniques, might influence the outcome of MHBO [8-10, 28-31]. In those previous meta-analyses of unilateral versus bilateral stenting for MHBO, some included both plastic and metal stents [8, 28, 29], and some included both percutaneous and endoscopic approaches [9, 29, 31]. Our present meta-analysis has some advantages over those previous meta-analyses: First, as we only included articles which used endoscopic metal stenting for MHBO, the risk of bias could be reduced. Second, the previous meta-analysis only pooled the stent dysfunction rates [9, 29-31]. However, our meta-analysis not only pooled the stent dysfunction rates, but also pooled the HR for stent patency. These results could evaluate the long-term outcomes in more detail. Third, subgroup analyses for the stent dysfunction rates were performed in this meta-analysis.

The limitations of our meta-analysis suggest several areas of potentially fruitful future research. First, most of the included studies were retrospective in nature, which may give rise to selection bias. Future RCTs will thus be needed to confirm or refute the findings outlined here. Secondly, the included studies used several different stent deployment techniques, principally stent-in-stent and side-by-side approaches, which can also bias the results. Thirdly, most of the included studies enrolled MHBO patients with a variety of cancers, which again can limit the wide applicability of the findings presented above.

\section{Conclusions}

Our meta-analysis demonstrated that endoscopic unilateral stenting had a higher technical success rate for MHBO patients than bilateral stenting. However, bilateral stenting could achieve longer stent patency.

\section{Conflict of interest}

The authors declare no conflict of interest.

\section{References}

1. Li J, Xiong Y, Yang G, et al. Complete laparoscopic radical resection of hilar cholangiocarcinoma: technical aspects and long- term results from a single center. Videosurgery Miniinv 2021; 16: $62-75$

2. Li M, Bai M, Qi X, et al. Percutaneous transhepatic biliary metal stent for malignant hilar obstruction: results and predictive factors for efficacy in 159 patients from a single center. Cardiovasc Intervent Radiol 2015; 38: 709-21.

3. Li M, Li K, Qi X, et al. Percutaneous transhepatic biliary stent implantation for obstructive jaundice of perihilar cholangiocarcinoma: a prospective study on predictors of stent patency and survival in 92 patients. J Vasc Interv Radiol 2016; 27: 1047-55.e2

4. Zhu HD, Guo JH, Huang M, et al. Irradiation stents vs. conventional metal stents for unresectable malignant biliary obstruction: a multicenter trial. J Hepatol 2018; 68: 970-7.

5. Perdue DG, Freeman ML, DiSario JA, et al. Plastic versus self-expanding metallic stents for malignant hilar biliary obstruction: a prospective multicenter observational cohort study. I Clin Gastroenterol 2008; 42: 1040-6.

6. Teng F, Xian YT, Lin J, et al. Comparison of unilateral with bilateral metal stenting for malignant hilar biliary obstruction. Surg Laparosc Endosc Percutan Tech 2019; 29: 43-8.

7. Hong WD, Chen XW, Wu WZ, et al. Metal versus plastic stents for malignant biliary obstruction: an update meta-analysis. Clin Res Hepatol Gastroenterol 2013; 37: 496-500.

8. Sawas T, Al Halabi S, Parsi MA, et al. Self-expandable metal stents versus plastic stents for malignant biliary obstruction: a meta-analysis. Gastrointest Endosc 2015; 82: 256-67.e7.

9. Ashat M, Arora S, Klair JS, et al. Bilateral vs unilateral placement of metal stents for inoperable high-grade hilar biliary strictures: a systemic review and meta-analysis. World J Gastroenterol 2019; 25: 5210-9.

10. Aghaie Meybodi M, Shakoor D, Nanavati J, et al. Unilateral versus bilateral endoscopic stenting in patients with unresectable malignant hilar obstruction: a systematic review and metaanalysis. Endosc Int Open 2020; 8: E281-90.

11. Naitoh I, Ohara H, Nakazawa T, et al. Unilateral versus bilateral endoscopic metal stenting for malignant hilar biliary obstruction. J Gastroenterol Hepatol 2009; 24: 552-7.

12. Iwano H, Ryozawa S, Ishigaki N, et al. Unilateral versus bilateral drainage using self-expandable metallic stent for unresectable hilar biliary obstruction. Dig Endosc 2011; 23: 43-8.

13. Liberato MJ, Canena JM. Endoscopic stenting for hilar cholangiocarcinoma: efficacy of unilateral and bilateral placement of plastic and metal stents in a retrospective review of $480 \mathrm{pa}$ tients. BMC Gastroenterol 2012; 12: 103.

14. Mukai T, Yasuda I, Nakashima M, et al. Metallic stents are more efficacious than plastic stents in unresectable malignant hilar biliary strictures: a randomized controlled trial. J Hepatobiliary Pancreat Sci 2013; 20: 214-22.

15. Hatamaru K, Yamashita Y, Uenoyama Y. The Study of endoscopic treatment for unresectable malignant hilar biliary obstruction, comparison of unilateral and bilateral drainage. Gastrointest Endosc 2017; 85: AB626.

16. Lee TH, Kim TH, Moon JH, et al. Bilateral versus unilateral placement of metal stents for inoperable high-grade malignant hilar biliary strictures: a multicenter, prospective, randomized study. Gastrointest Endosc 2017; 86: 817-27. 
17. Xia MX, Cai XB, Pan YL, et al. Optimal stent placement strategy for malignant hilar biliary obstruction: a large multicenter parallel study. Gastrointest Endosc 2020; 91: 1117-28.e9.

18. Staub J, Siddiqui A, Murphy M, et al. Unilateral versus bilateral hilar stents for the treatment of cholangiocarcinoma: a multicenter international study. Ann Gastroenterol 2020; 33: 202-9.

19. Higgins JP, Altman DG, Gøtzsche PC, et al. The Cochrane Collaboration's tool for assessing risk of bias in randomised trials. BMJ 2011; 343: d5928.

20. Lo CK, Mertz D, Loeb M. Newcastle-Ottawa Scale: comparing reviewers' to authors' assessments. BMC Med Res Methodol 2014; 14: 45.

21. Fu YF, Zhou WJ, Shi YB, et al. Percutaneous stenting for malignant hilar biliary obstruction: a randomized controlled trial of unilateral versus bilateral stenting. Abdom Radiol 2019; 44: 2900-8.

22. Yin X, Li DM, Yang F, et al. Self-expanded metallic stent insertion for hilar cholangiocarcinoma: comparison of unilateral and bilateral stenting. J Laparoendosc Adv Surg Tech A 2019; 29: 1501-6.

23. Chang G, Xia FF, Li HF, et al. Unilateral versus bilateral stent insertion for malignant hilar biliary obstruction. Abdom Radiol 2017; 42: 2745-51.

24. Gwon DI, Ko GY, Sung KB, et al. Percutaneous biliary metallic stent placement in patients with unilobar portal vein occlusion caused by advanced hilar malignancy: outcome of unilateral versus bilateral stenting. AJR Am J Roentgenol 2011; 197: 795-801.

25. Rerknimitr R, Angsuwatcharakon P, Ratanachuek T, et al. Asia-Pacific consensus recommendations for endoscopic and interventional management of hilar cholangiocarcinoma. J Gastroenterol Hepatol 2013; 28: 593-607.

26. Naitoh I, Hayashi K, Nakazawa T, et al. Side-by-side versus stent-in-stent deployment in bilateral endoscopic metal stenting for malignant hilar biliary obstruction. Dig Dis Sci 2012; 57: 3279-85.

27. Li TF, Chen C, Han XW, et al. Clinical efficacy of metallic biliary stents combined with different anti-cancer treatments in the management of bile duct cancer. Hepatogastroenterology 2014; 61: 22-6.

28. Hong W, Sun X, Zhu Q. Endoscopic stenting for malignant hilar biliary obstruction: should it be metal or plastic and unilateral or bilateral? Eur J Gastroenterol Hepatol 2013; 25: 1105-2.

29. Li M, Wu W, Yin Z, et al. Unilateral versus bilateral biliary drainage for malignant hilar obstruction: a systematic review and meta-analysis. Zhonghua Gan Zang Bing Za Zhi 2015; 23: $118-23$.

30. Fu YF, Xu YS, Shi YB, et al. Percutaneous metal stenting for malignant hilar biliary obstruction: a systematic review and meta-analysis of unilateral versus bilateral stenting. Abdom Radiol 2021; 46: 749-56.

31. Chen ZK, Zhang W, Xu YS, et al. Unilateral versus side-by-side metal stenting for malignant hilar biliary obstruction: a metaanalysis. J Laparoendosc Adv Surg Tech A 2021; 31: 203-9.

Received: 8.12.2020, accepted: 11.01.2021. 